\title{
The estimation of the interrelation between paleoclimatic time series
}

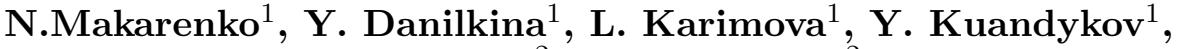 \\ M. Eronen ${ }^{2}$ and S. Helama ${ }^{2}$ \\ ${ }^{1}$ Institute of Mathematics, Pushkin str. 125, 480100, Almaty, Kazakhstan \\ email: chaos@math.kz \\ ${ }^{2}$ Department of Geology, PO Box 64, University of Helsinki, Finland \\ email: helama@cc.helsinki.fi
}

\begin{abstract}
The work presents the nonlinear interrelation estimation between paleoclimatic time series using reconstructions of topological models obtained by embedding corresponding time series into $R^{n}$
\end{abstract}

Variability of natural processes over the North Atlantic sector, including coupled oceanatmosphere system, has extensive climate impacts from eastern seaboard of the United States to Siberia, from the Arctic to the subtropical Atlantic, see Hurrel et al. (2001). Variations in the climate over Greenland and North Europe are likewise largely dictated by this natural circulation system (Barlow et al. (1997)). Understanding of the connections of the climate perturbations in different regions requires long time series of paleoclimatic proxy records. Such records of temperature variability within the North Atlantic sector have been produced by isotopic measurements of ice-cores and tree-ring chronologies of living and subfossil wood, in Greenland and North-West Europe, respectively. Joint investigation of these records, as well as further development of synoptic scale paleoclimatology in Greenland and adjacent areas, should improve the understanding of the climatic variability over the entire North Atlantic sector (Barlow et al. (1997)).

Hence, for a proper reconstruction of the paleoclimate it is very important to estimate the interrelation between different proxy data. Since paleoclimatic proxy records are superposition of many uncontrollable natural processes and consequently structured data, we investigate proxy data with the help of topological dynamic methods to reveal their interrelationship, see Sauer et al. (1991).

Linear relation between nonlinear time series can be absent or very weak. So we use the nonlinear correlation estimation method (Čenis et al. (1991)), the idea of which lies in the following. The reconstruction of each time series is made by means of the Takens algorithm in Euclidean space of appropriate dimension (Sauer et al. (1991)), and will be denoted as rec $X$ and rec $Y$. Then, for reconstruction rec $X$ the number of vector pairs, having distances equal or less than some positive value $\varepsilon$, is calculated. Next, the number of $\varepsilon$-close vectors of model rec $Y$ is estimated basing on the condition that their synchronous analogs in rec $X$ are also $\varepsilon$-close. If the statistical relation between $\left\{x_{i}\right\}$, and $\left\{y_{i}\right\}$ does exist, then it will take place for models rec $X$ and rec $Y$ also. In this case, the statistics will depend on parameter $\varepsilon$. On the contrary, such a dependence is absent, if the models (and the time series) are statistically independent. Formally, in order to estimate a power of interrelations, the cross correlation ratio is used

$$
K_{x y}(\varepsilon)=\sqrt{\frac{\sum_{i \neq j}\|y(i)-y(j)\|^{2} \Theta(\varepsilon-\|x(i)-x(j)\|)}{\sum_{i \neq j} \Theta(\varepsilon-\|x(i)-x(j)\|)}},
$$




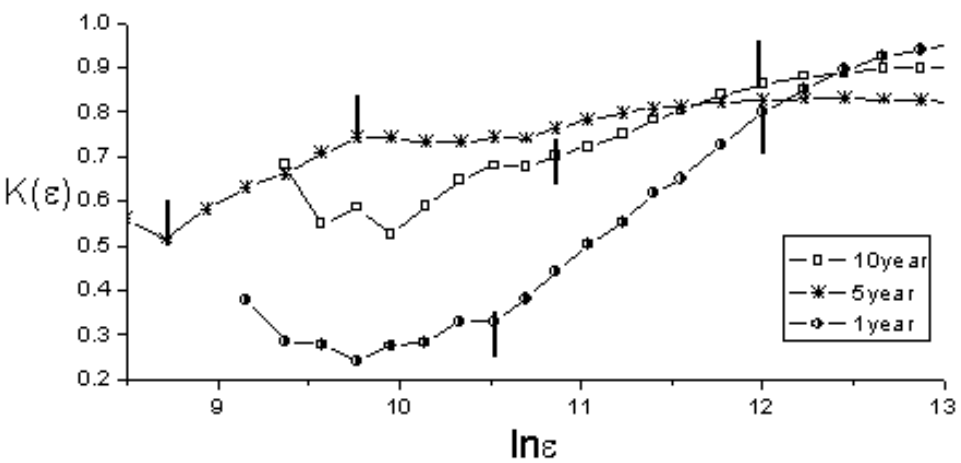

Figure 1. Estimations of nonlinear interrelation between temperature and oxygen isotope ratio time series for different time scales

where $x(i)=\left\{x_{i}, x_{i+\tau}, \ldots, x_{i+(d-1) \tau}\right\}$ and $y(j)=\left\{y_{j}, y_{j+\tau}, \ldots, y_{j+(d-1) \tau}\right\}$ are delay vectors of rec $X$ and rec $Y$ systems, $d$ is embedding dimension $\tau$ is a lag and $\Theta$ is Heaviside function. If systems $X$ and $Y$ are related then one can expect that $\|x(i)-x(j)\|<\varepsilon \Rightarrow$ $\|y(i)-y(j)\| \approx \varepsilon$. If it is not so, $K_{x y}$ does not depend on $\varepsilon$. As a rule the graph of $K_{x y}$ versus $\log \varepsilon$ is used and rough estimation may be found by means of a slope value $s=K_{x y} / \log (\varepsilon)$.

The interdependence of two time series that are proxy indicators of climate fluctuations on large time scales have been researched. The first time series is the yearly average July temperature in North Lapland from 5510 BP to 1993 AD (Eronen et al. (2002)). The second time series is the abundance ratio of oxygen isotope in ice cores of Greenland from 8065 BP to 1987 AD (Stuiver et al. (1995)). As the system $X$, a topological reconstruction of temperature time series obtained from tree-ring data is taken. The $Y$ system is a topological reconstruction from oxygen isotope time series. To obtain significant results, denoising and smoothing procedures have been applied. To investigate interrelation on different time scales, from the original annual time series we obtained 5 -years and 10years sampled time series. Evaluated dependencies of $K$ versus $\ln (\varepsilon)$ are demonstrated in Figure 1. The slope of the graph linear intervals enclosed between two vertical lines confirms the presence of the interdependence between $X$ and $Y$ time series. The largest interdependence between $X$ and $Y$ has been found for the annual time series and results in $s=0.34$. The next values $s=0.23$ and $s=0.13$ were estimated for the 5 -year and 10-year time series correspondingly.

\section{Acknowledgements}

The support from INTAS grant N2001-0550 greatfully acknowledged.

\section{References}

Hurrell, J. W., Kushnir, Y., Visbeck, M. 2001, Science 291(5504), p. 603

Barlow, L., Rogers, J., Serreze, M., Barry, R. G. 1997, J.Geophys.Res. 102(C12), p. 26333

Sauer, T., Yorke, J.A., Casdagli, M. 1991, J.Stat.Phys. 65, p. 579

Čenis, A., Lasiene, G., Pyragas, K. 1991 Physica D, 52, p. 332

Eronen, M., Zetterberg, P., Briffa, K., Lindholm, M., Merilainen, J., Timonen, M. 2002 Holocene 12(6), p. 673

Stuiver M., Grootes P., Braziunas F. 1995 Quat.Res. 44, p. 341 